\title{
THE APRIL MEETING IN NEW YORK
}

The six hundred first meeting of the American Mathematical Society was held from Monday, April 29 through Thursday, May 2 at the Hotel New Yorker. There were 570 persons registered, including 426 members of the Society.

The Committee to select Hour Speakers for Eastern Sectional Meetings arranged two invited addresses. Professor H. E. Rauch of Yeshiva University spoke on Monday afternoon on $A$ transcendental view of the space of algebraic Riemann surfaces. Professor G. A. Hedlund introduced the speaker. On Tuesday morning, Professor G. Shimura of Princeton University gave an address entitled Arithmetic of unitary groups and its applications. Professor G. W. Mostow introduced the speaker.

On Wednesday afternoon there was a Special Invited Address by Academician Sergei Sobolev. His title was Approximate integration on a sphere. Dr. J. P. La Salle presided at the address and introduced the speaker. Academician Sobolev is the Director of the Institute of Mathematics at Novosibirsk, U.S.S.R. and was in the United States for a three week period as a Visiting Lecturer under the sponsorship of the Society. The program of Visiting Lecturers is supported by the National Science Foundation.

There was a Symposium on Stochastic Processes in Mathematical Physics and Engineering on Tuesday afternoon and on Wednesday and Thursday. The Invitations and Organizing Committee, responsible for the program of the Symposium, consisted of Richard Bellman, A. T. Bharucha-Reid, Marc Kac, J. M. Richardson, J. B. Keller, Lotfi Zadeh, and David Slepian. Their efforts resulted in a program of fifteen invited addresses. Publication of the papers of the Symposium has been authorized as Volume XVI of the series Proceedings of Symposia in Applied Mathematics.

On Monday and Tuesday morning, there were nine sessions for contributed papers, at which about sixty papers were presented. The presiding officers at these sessions included Professors J. B. Bartoo, V. F. Cowling, C. C. Hsiung, Mr. A. E. Hurd, Professor B. L. Reinhart, Dr. Samuel Schechter, Professors H. W. E. Schwerdtfeger, G. C. Webber and P. K. Wong.

The Council met on Monday, April 29, 1963, at 7:00 P.M.

The Secretary announced the election of the following three hundred forty-one persons to ordinary membership in the Society:

Mr. Zuhdi A. Abdo, De Paul University;

Miss Thelma L. Abell, Hammond High School, Hammond, Indiana; 
Mr. Charles H. Ackerknecht, Los Angeles City School District, Los Angeles, Calif.; Professor Furio Alberti, University of Illinois;

Mr. Brian R. Alspach, University of California, Santa Barbara;

Mr. Ronald M. Anderson, Collins Radio Co., Cedar Rapids, Iowa;

Mr. Dang D. Ang, University of Saigon, Saigon, Vietnam;

Mr. Lawrence J. Arnold, Occidental College;

Mr. Pasquale J. Arpaia, Clarkson College;

Professor David A. Bachrach, State University of New York at New Paltz;

Professor Richard H. Balomenos, University of New Hampshire;

Mr. Samuel E. Barger, College of St. Thomas, St. Paul, Minnesota;

Mr. Thomas V. Baucom, State Teachers College, Frostburg, Maryland;

Mr. Oliver G. Becker, I.T.T. Communication Systems Inc., Paramus, New Jersey;

Mr. John J. Benedetto, University of Toronto, Toronto, Canada;

Mr. Daniel D. Benice, University of Buffalo;

Mr. Bruce S. Berger, Vanderbilt University;

Mr. Harry W. Berkowitz, Drexel Institute of Technology;

Mr. Georges L. Berlioz, Oakland, California;

Mr. Herbert J. Bernstein, Washington Square College, New York University;

Mr. Paul M. Berry, University of Oklahoma;

Professor David R. Beuerman, Erie County Technical Institute;

Mr. Edward C. Bittner, National Aeronautics and Space Administration, Cleveland, Ohio;

Mr. Beresford M. Bland, University of Southampton, Southampton, England;

Mr. Barry W. Boehm, Rand Corporation, Santa Monica, California;

Professor Mario Borelli, University of British Columbia;

Mr. Thomas Boyd, Lockwood, Kessler and Bartlett, Inc., Syosset, New York;

Mr. Alan U. Brender, University of California, Berkeley;

Mr. Jacob L. Bricker, Hughes Aircraft Co., Fullerton, California;

Mr. James E. Bright, U. S. Army, New York, New York;

Mr. William E. Brodie, Paine College;

Mrs. Adelaide J. Brooks, Harris Public Schools, Harris, Oklahoma;

Professor Helen L. Brooks, University of Toledo;

Mr. Walter J. Bruecks, University of Pennsylvania;

Mr. Vincent J. Bruno, San Francisco State College;

Mr. Steven J. Bryant, University of California, Berkeley;

Mr. Colin W. Bryer, National Research Institute of Mathematical Sciences, Pretoria, South Africa;

Mr. Larry L. Bumgarner, Oak Ridge National Laboratories, Oak Ridge, Tennessee;

Mr. Barry J. Bunow, University of California, Berkeley;

Professor Edgar H. Buyco, St. Mary's University, Nova Scotia;

Mr. George D. Byrne, Iowa State University;

Mr. Thomas E. Caldwell, C-E-I-R, Inc., Fort Huachuca, Arizona;

Mr. Richard L. Campbell, Case Institute of Technology;

Professor David S. Carter, Oregon State University;

Professor Geoffrey B. Charlesworth, Hofstra College;

Mr. Isaac Chavel, Brooklyn College;

Mr. John G. Christiano, Northern Illinois University;

Professor Richard A. Cleveland, University of British Columbia;

Mr. John D. Cloud, North American Aviation, Torrance, California;

Mr. Edward M. Cohen, University of Massachusetts; 
Mr. Jeffrey M. Cohen, Yale University;

Mr. Charles A. Combs, Jr., General Electric Co., Phoenix, Arizona;

Mr. Dale R. Comstock, Oregon State University;

Mr. Andrew Conti, Youngstown University;

Mr. Edward D. Conway III, Indiana University;

Mr. Thurlow A. Cook, University of Buffalo;

Mr. Bernard Courteau, Universite de Montreal, Montreal, Quebec, Canada;

Mr. George E. Crane, Lockheed Missiles and Space Co., Palo Alto, California;

Mr. Larry J. Cummings, De Vry Technical Institute;

Professor George S. Cunningham, University of Maine;

Professor J. Dwight Daugherty, Kutztown State College;

Professor Budmon R. Davis, University of Toledo;

Mr. Paul L. Davis, West Virginia University;

Mr. William J. Davis, Case Institute of Technology;

Mr. Henry P. Decell, Jr., Louisiana State University;

Mr. Donald J. Deckard, Humble Oil and Refining Co., Houston 1, Texas;

Mr. David L. Deever, Ohio State University;

Mr. John L. Denny, Jr., Indiana University;

Mr. Charles A. Desoer, University of California, Berkeley;

Mr. Lawrence George Deysach, Harvard University;

Mr. James M. Dickey, University of Michigan;

Professor Edwin L. Dolney, Eastern Montana College;

Mr. Bernard M. Donahoe, National Aeronautics and Space Administration, Santa Monica, California;

Professor Ronald G. Douglas, University of Michigan;

Professor John P. Downes, State University College at Oneonta;

Professor James R. Downing, Illinois State Normal University;

Professor Mitrovic Dragisa, Faculty of Technology, Zagreb, Yugoslavia;

Mr. Bruce A. Drew, The Pillsbury Co., Minneapolis, Minnesota;

Mr. Morton R. Dubman, Rocketdyne, Canoga Park, California;

Mrs. Estelle C. Dunlap, MacFarland Junior High School, Washington, D. C.;

Professor Marguerite E. Dunton, Sacramento State College;

Mr. Delmar A. Dyreson, New Mexico Highlands University;

Mr. Thomas E. Easley, Philco Western Dev. Labs., Palo Alto, California;

Mr. Howard L. Egan, Washington University;

Mr. John Eglitis, Kansas City 13, Missouri;

Mrs. Eleanor E. Ehrlich, Schenectady 9, New York;

Mr. Isidore Eisenberger, Jet Propulsion Lab., Pasadena, California;

Mr. David L. Elliott, U. S. Naval Ordnance Test Station, Pasadena, California;

Mr. William R. Emerson, University of California, Berkeley;

Mr. Arthur B. Evans, Ginn and Company, Boston 17, Massachusetts;

Miss Elinor R. Evenchick, University of Maryland;

Mr. Robert J. Fabian, Case Institute of Technology;

Mr. Charles G. Fain, Technical Operations Incorporated, Burlington, Massachusetts;

Mr. William W. Fairchild, University of Pennsylvania;

Miss Ruth L. Faith, Melpar Incorporated, Falls Church, Virginia;

Professor Henryk Fast, University of Notre Dame;

Dr. Alvin E. Fein, Westinghouse Electric Corp., Baltimore, Maryland;

Professor Virginia I. Felder, University of Southern Mississippi;

Mr. Heinz A. Fiedler, University of Utah;

Mr. Harvey J. Finberg, Adelphi College; 
Mr. E. Arthur Fiser, Oxford, Ohio;

Mr. Edward R. Fisher, Jr., Carnegie Institute of Technology;

Mr. Robert A. Fisher, United States Army;

Mr. Charles E. Flagg, Kimball Union Academy;

Professor William T. Fletcher, LeMoyne College;

Mr. David Fowler, University of Nebraska;

Professor James W. Frankowsky, Lincoln University;

Mr. Brad C. Frederic, General Electric TEMPO, Santa Barbara, California;

Mr. Thomas A. Fredericks, University of Texas;

Mr. R. Don Freeman, Jr., Massachusetts Institute of Technology;

Mr. H. Stewart French, Chrysler Corporation, Detroit, Michigan;

Mrs. Marcelle B. Friedman, New York University;

Mr. Nathaniel Friedman, Brown University;

Mr. Francis R. Frola, North American Aviation Inc., Downey, California;

Mr. Clinton P. Fuelling, United States Air Force, Arlington, Virginia;

Mr. Derek J. H. Fuller, Pius XII University College, Africa;

Mr. Donald G. Fuller, Northern Illinois University;

Mr. Daniel Gallin, University of California, Berkeley;

Professor Theodor Ganea, Purdue University;

Mr. Peter J. A. Gaposchkin, University of California, Berkeley;

Lt. Col. Joseph S. Gardner, Missouri Military Academy;

Mr. Ladnor D. Geissinger, Indiana University;

Professor Harvey M. Gelder, Western Washington State College;

Mr. Michael S. Geller, University of Manitoba, Winnipeg, Manitoba, Canada;

Mr. Michael C. Gemignani, University of Notre Dame;

Mr. Subhas C. Ghorai, Michigan State University;

Mr. Frank H. Gille, American Data Processing Incorporated, Grosse Pointe Woods, Michigan;

Miss Erna Gold, Columbia University;

Mr. Nathan L. Gordon, Radio Corporation of America Laboratories, Princeton, N. J.;

Mr. Saul B. Gorski, Lawrence Radiation Lab., Livermore, California;

Mr. Stephen R. Graczyk, Cornell Aero Lab., Inc., Buffalo 21, New York;

Mr. Kenneth L. Grant, Indiana University;

Mr. Eugene R. Grassler, Mitra Corporation, Bedford, Massachusetts;

Mr. Billey G. Gray, University of Texas;

Professor Frederick R. Gray, Prairie View A. and M. College;

Mr. Juan Grimberg, Aerospace Corporation, Los Angeles 45, California;

Mr. Shlomo Halfin, University of California, Berkeley;

Mr. Wallace L. Hamilton, University of Utah;

Mr. Randall R. Harris, Lincoln Junior College;

Mr. Richard G. Herrmann, General Electric, Santa Barbara, California;

Professor George W. Heigho, Suffolk University;

Professor Francis L. Higman, Niagara University;

Mr. Arthur L. Hilt, General Electric Co., King of Prussia, Pennsylvania;

Mr. Julius H. Hlavaty, New Rochelle, New York;

Mr. Anthony E. Hoffman, University of Nebraska;

Miss Ruth I. Hoffman, Denver Schools, Denver, Colorado;

Professor Antony S. Holland, University of Alberta;

Mr. Pui-Lam Hon, Hong Kong Government, Hong Kong

Mr. Fred Hoofer, Ridgewood High School, Norridge 34, Illinois;

Mr. Norman Hosay, University of Wisconsin; 
Professor Wei Hsuan, Ithaca College;

Mr. Robert E. Huddleston, Texas Christian University; Professor Frank N. Huggins, A. and M. College of Texas;

Mr. Albert E. Hurd, Massachusetts Institute of Technology;

Professor Syed Q. Husain, Rajshahi University, Rajshahi, East Pakistan;

Mr. Allen R. Hyde, Ronald Press Co., New York 10, New York;

Professor Donald L. Iglehart, Cornell University;

Mr. Harvey Jackins, Personal Counselors Inc., Seattle, Washington;

Mr. Leo F. Jarzomb, Collins Radio Co., Newport Beach, California;

Mr. Barry E. Johnson, Yale University;

Mr. Jimmie L. Johnson, University Park, New Mexico;

Professor Johnny R. Johnson, Appalachian State Teachers College;

Mr. Neil W. Johnson, Computer Associates, Inc., Woburn, Massachusetts;

Mr. Bradley M. Johnston, Lawrence Radiation Laboratory, Livermore, California;

Mr. Geoffrey T. Jones, Australasian Missionary College, Australia;

Professor Wilbur J. Jonsson, University of Manitoba;

Mr. Wayne J. Jurgensen, San Marcos School;

Mr. Jay Kadashaw, Wayne State University;

Mr. Hermann F. Karreman, Princeton University;

Mr. Paul Katz, University of California, Los Angeles;

Mr. Robert G. Kayel, Grumman Aircraft Engineering Corporation, Bethpage, N. Y.;

Mr. John G. Kennell, Pleasant Corporation, Pt. Pleasant, New Jersey;

Miss Anne M. Keoning, Manhattanville College;

Professor Angus Kerr-Lawson, University of Waterloo;

Mr. Robert E. Kibler, University of Chicago;

Professor Fred Kiokemeister, Mount Holyoke College;

Mr. George C. Killough, University of Tennessee;

Professor Nand Kilshore, University of San Francisco;

Mr. Jerome R. Kirk, Johns Hopkins University;

Mr. William J. Klein, North American Aviation, Downey, California;

Professor H. Kleisli, University of Ottawa, Ontario, Canada;

Miss Genevieve M. Knight, Edison Junior College;

Miss Patricia A. Knoop, National Aeronautics and Space Administration, Cleveland, Ohio;

Mr. Stanley M. Kolsan, De Vry Technical Institute;

Mr. Leon Kotin, U. S. Army Electronics R. and D. Laboratory, Ft. Monmouth, N. J.;

Rev. James P. Kramper, Creighton University;

Professor Howard E. Krehbiel, Iowa Wesleyan College;

Professor Melven R. Krom, University of California, Davis;

Professor Radha G. Laha, Catholic University of America;

Mr. Gary B. Laison, Lehigh University;

Mr. Howard A. Larson, Phillips Petroleum Co., Idaho Falls, Idaho;

Mr. Loren C. Larson, University of Kansas;

Mr. James A. Lavita, New York University;

Mr. Edgar S. Lavoie, Police Academy, New York, New York;

Mr. Robert W. Lawler, Boeing Co., New Orleans, Louisiana;

Professor Phillip E. Leahy, St. John's University;

Professor Teddy C. J. Leavitt, State University College, Plattsburgh, New York;

Mr. F. Treville Leger, Ginn and Company, Boston 17, Massachusetts;

Mr. John E. Lehnsen, American Cyanamid Co., Stamford, Connecticut;

Mr. William W. Leonard, University of South Carolina; 
Mr. David M. Levin, Lowell High School, San Francisco, California;

Mr. James E. L'Heureux, Fox Valley Center, University of Wisconsin, Menasha, Wisconsin;

Mr. Yuling Li, Harvard Medical School;

Mr. Dale W. Lick, University of Redlands;

Dr. Julius Lieblein, U. S. Navy Department, Washington, D. C.;

Mr. You-Feng Lin, Tulane University;

Professor Charles M. Lindsay, Coe College;

Mrs. Jeannette E. Lockley, Oakland City College;

Mr. Donald A. Lutz, Syracuse University;

Mrs. F. Jessie MacWilliams, Bell Telephone Laboratories, Holmdel, New Jersey;

Mr. Hagzl-Rao V. Madi-Raj, University of Keele, North Staffordshire, England;

Mr. Spyridon S. Magliveras, University of Florida;

Mrs. Lucille Maier, Rosary Hill College;

Miss Coline M. Makepeace, Hood College;

Mrs. Ethel C. Marden, National Bureau of Standards, Washington, D. C.;

Mr. Frank J. Markovich, General Electric, Santa Barbara, California;

Professor Herman B. Marks, U. S. Naval Postgraduate School, Monterey, California;

Mr. Charles D. Marshall, University of Kansas;

Mr. Eugene V. Martin, Ohio State University;

Miss Hosna Massouda, American Mathematical Society, Providence, Rhode Island;

Mr. Donald E. Maurer, University of Colorado;

Mr. Emory C. McClinton, Atlanta 18, Georgia;

Mr. Arthur W. McIver, Laboratory for Electronics, Monterey, California;

Professor Earl H. McKinney, Ball State Teachers College;

Mr. Arthur J. McMahon, Rhode Island Department of Education, Providence, Rhode Island;

Professor Hugh D. McNiven, University of California, Berkeley;

Mr. James Leonidas Michael, Datatrol Corporation, Silver Spring, Maryland;

Professor Francisco Migliore, Villanova University;

Mr. Francis L. Miksa, Illinois Bell Telephone Co., Aurora, Illinois;

Mr. Frederick J. Milford, Battelle Memorial Institute;

Mr. Charles E. Miller, Jr., Radio Corporation of America, Cambridge, Ohio;

Mr. Ernest H. Milton, Jr., University of Utah;

Capt. Robert B. Mollo, Ann Arbor, Michigan;

Professor Joseph M. Moser, San Diego State College;

Mr. Stewart F. Musket, Armour Research Foundation, Chicago, Illinois;

Mr. Weston I. Nathanson, Douglas Aircraft Co., Culver City, California;

Dr. Israel Navot, Technion, Israel Institute of Technology, Haifa, Israel;

Father Joseph M. Nebesky, Cathedral College Preparatory;

Mr. Allyn H. Nelson, Longwood High School, Middle Island, New York;

Mr. Eric D. Nix, Random House, Inc., New York, New York;

Mr. Arthur A. Noma, Army Map Service, Washington, D. C.;

Mr. John W. Norton, Union Oil Co. of California, Los Angeles, California;

Miss Gladys A. O'Brien, Chicago Board of Education, Chicago, Illinois;

Mr. Stephen O'Brien, University College;

Professor Anthony G. Oettinger, Harvard University;

Mr. Brian J. O'Mahoney, Cambridge University;

Mr. Raymond E. Orth, Jr., Aerospace Corporation, El Segundo, California;

Mr. Bernard H. Paiewonsky, Aeronautical Research Associates, Princeton, N. J.;

Mr. Samuel Hugh Paine, Jr., De Vry Technical Institute; 
Mr. James A. Painter, International Business Machines Corporation, Poughkeepsie, New York;

Mr. Donald B. Parker, Mohawk Valley Technical Institute;

Mrs. Lucille H. Parks, University of Arizona;

Mr. John Passanante, System Development Corporation, Corvallis, Oregon;

Mr. Richard L. Patterson, University of Michigan;

Professor Oliver W. Peterson, Eastern Montana College of Education, Montana;

Mr. Demetrios J. Poros, Air Force Cambridge Research Laboratory, Bedford, Mass.;

Mr. Willis B. Porter, Iberia Parish School Board, New Iberia, Louisiana;

Lt. Calvin J. Powell, Jr., Fort Bliss, Texas;

Mr. James R. Price, University of Illinois;

Mr. Eugene H. Primoff, New York University;

Mr. Gudrun G. Quenzler, New Haven, Connecticut;

Professor James F. Ramaley, University of California, Berkeley;

Mr. William C. Ramaley, University of Colorado;

Mr. Joseph S. Romanow, Massachusetts Institute of Technology;

Professor Richard W. Reichhardt, Marquette University;

Mrs. Mary O. Reister, The Franklin Institute;

Mr. Henry R. Richardson, Brown University;

Professor Sara L. Ripy, Agnes Scott College;

Mr. James B. Robertson, Indiana University;

Mr. Staman F. Rodfong, Case Institute of Technology;

Mr. John F. Rogers, Berwick High School, Berwick, Louisiana;

Mrs. Hannah W. Rosenbaum, New York University;

Mr. Mitchell J. Rossin, Pennsylvania State University;

Mr. Bernard Russo, University of California, Los Angeles;

Professor Jagdish S. Rustagi, University of Cincinnati;

Mr. Jacob C. Sabto-Agami, Bell Telephone Laboratories, Murray Hill, N. J.;

Mr. Jerry D. St. Clair, North American Aviation, Downey, California;

Mr. Gerald M. Samson, Flint Community Junior College, Flint, Michigan;

Mr. Leroy Sathre, North American Aviation, Anaheim, California;

Mr. Roy G. Sawtell, Jr., South San Francisco High School, South San Francisco, California;

Mr. Willy Schild, International Electric Corporation, Paramus, New Jersey;

Miss Diane Schlieckert, Syracuse University;

Mr. Paul S. Schnare, Louisiana State University in New Orleans;

Mr. Richard M. Schori, State University of Iowa;

Mr. Verne Schwab, Applied Physics Laboratory, Silver Spring, Maryland;

Mr. John D. Scougale, Standard Oil Company, Cleveland, Ohio;

Mr. James D. Sebastian, Wisconsin State College;

Mr. John J. Sember, University of Massachusetts;

Mr. George Senge, University of California, Los Angeles;

Mr. Lawrence C. Shepley, Princeton University;

Lt. Richard B. Sher, University of Utah;

Professor Sakai Shoichiro, Yale University;

Mr. David W. Showalter, University of North Carolina;

Mr. James L. Sieber, Pennsylvania State University;

Mrs. Carole H. Sirovich, New York University;

Sister Gregory Mari, The Cardinal Stritch College;

Sister Mary Neal Moran, Mundelein College;

Mr. Julius Smith, University of Illinois; 
Mr. Donald R. Sondergold, Connecticut General Life Insurance Co., Hartford, Connecticut;

Professor W. Allen Spivey, University of Michigan;

Mr. Olaf P. Stackelberg, University of Minnesota;

Mr. David E. Stahl, Computer Concepts, Inc., Washington, D. C.;

Mr. Selig Starr, Army Research Office, Arlington, Virginia;

Mr. Michael J. Sullivan, Illinois Institute of Technology;

Professor Selma F. Sysal, Massachusetts Institute of Technology;

Mr. Emery J. Szmrecsanyi, Wayne State University;

Mr. Nazih J. Taleb, Dar Al-Handash, Beirut, Lebanon;

Mr. Mark G. Tanenbaum, Massachusetts Institute of Technology;

Professor D. V. Thampuran, University of Florida;

Mr. James W. Thatcher, University of Michigan;

Mr. Edward H. Theil, New York University;

Mr. Melvin C. Thornton, University of Illinois;

Professor Donald H. Trahan, University of Pittsburgh;

Mr. Dennis Travis, Columbia University;

Mr. Marvin D. Tretkoff, New York University;

Mr. Dudley R. Urgan, Altadena, California;

Mr. Charles A. Vok, Rocketdyne, Canoga Park, California;

Major Henry Leon Walker, Scott Air Force Base, Illinois;

Mr. William A. Walker, Naval Ordnance Laboratory, White Oak, Maryland;

Mr. Joseph Warren, Salem State College;

Mr. Howard C. Wasserman, University of Pennsylvania;

Mr. Alexander Weiner, Hofstra University;

Mr. Lee G. Wentling, Jr., Research Analysis Corporation, Bethesda, Maryland;

Mrs. Virginia W. Whiteley, University Park, New Mexico;

Mr. Raymond E. Whitney, Pennsylvania State University;

Professor Robert A. Wijsman, University of Illinois;

Professor Peter Wilker, University of Kansas;

Mr. Stanley G. Williamson, University of California, Santa Barbara;

Mr. Ralph P. Winter, Minneapolis-Honeywell Regulator Co., Hopkins, Minnesota;

Mr. James R. Wohlever, Iona College;

Miss Catherine E. Wormley, Colonie Central High School, Albany, New York;

Mr. John M. Worrell, Jr., Sandia Corporation, Sandia Base, New Mexico;

Mr. Roy E. Worth, University of Georgia;

Mr. Bostwick F. Wyman, University of California, Berkeley;

Mr. Paul R. Young, Massachusetts Institute of Technology;

Mr. Charles T. Zahn, Jr., University of Wisconsin;

Mr. Eli A. Zeitlin, China Lake, California;

Mrs. Mary H. Zink, Elmhurst College;

Professor Paul R. Zuckerman, New York University,

The Secretary announced that the following had been admitted to the Society in accordance with reciprocity agreements with various mathematical organizations:

Wiskundig Genootschap te Amsterdam: Mr. Jan Christoph Boland, Mr. Herman Gerrit Brinkman, Mr. Philip Van Elteren, Professor Gerhardus H. A. Grosheide, Dr. Antonie F. Monna, Mr. Pieter Mullender, Mr. Hans R. Zweerus.

Societe Mathematique de Belgique: Professor Pierre Wuyts. 
Calcutta Mathematical Society: Mr. Subhendu K. Datta.

Chinese Mathematical Society: Mr. Hsu-Tung. Ku.

Deutsche Mathematiker Vereinigung: Mr. Walter Felscher, Professor Nikolaus Hofreiter, Professor Heinz Kunle, Dr. Herbert Naumann, Dr. Gerhard J. Neubauer.

Edinburgh Mathematical Society: Mr. David John Simms.

Societe Mathematique de France: Mr. Manuel A. Chaves, Mr. Maurice Glaymann, Miss Paulette L. Libermann, Mr. Nicholas Minorsky, Professor Louis E. Perrin, Mr. Julian Petrescu, Mr. Raymond Queneau.

Indian Mathematical Society: Mr. Chaitan P. Gupta, Dr. Mysore N. L. Narasimhan, Dr. Qazi I. Rahman, Professor Jonnavithula Ramakanth, Profesor Subhash C. Saxena.

Union Matematica Italiana: Professor Mario Benedicty, Professor Edmondo Morgantini.

Israel Mathematical Union: Professor Israel Berstein, Mr. Abraham Ginzburg, Dr. Amram Meir.

Mathematical Society of Japan: Professor Atuso Fujimoto, Professor Akio Hattori, Mr. Yoshio Hayashi, Mr. Teruo Ikebe, Mr. Takeshi Kotake, Professor Atuo Komatu, Professor Takashi Nagano, Professor Nobuo Nobusawa, Professor Kaoru Sekino, Professor Goro Shimura, Mr. Tseneyo Yamanoshita.

London Mathematical Society: Mr. Francis A. J. Ford, Mr. William V. D. Hodge, Mr. Albert C. Offord, Mr. John S. Shepherdson, Dr. John A. H. Shepperd, Mr. Edward C. Thompson, Dr. Eric W. Wallace.

Malayan Mathematical Society: Professor Christie J. Elizer.

Polskie Towarzystwo Matematyczne: Mr. Czestaw M. Bessaga, Mr. Andrzej Granas, Professor Karol Sieklucki.

Sociedade de Mathematica de São Paulo: Professor Waldyr M. Oliva, Mr. Nelson Onuchic, Mr. Renzo Angelo Antonio Piccinini.

Schweizersche Mathematische Gesellschaft: Professor Christian Blatter, Professor Hans H. Keller, Mr. Philippe M. Tondeur.

It was reported that five hundred twenty-two persons were elected to membership in the Society on nomination of institutional members as indicated:

Adelphi College: Mr. Dennis Eisen, Mr. Walter C. Lebensohn, Mr. Michael A. Orleck. University of Alabama: Mrs. Janet S. Allsbrook, Mr. David W. Murrell, Jr., Professor Gaston Smith.

Alameda County State College: Mr. David L. Durbin, Mr. Ira W. Morrison, Mrs. Helene B. Schrader.

University of Alberta: Mr. Richard J. Mansfield, Miss Bente G. Rasmussen.

Andrews University: Mr. Watson L. Chin, Mr. Cecil E. Davis, Mr. Milton S. Hwang. Arizona State University: Mr. Charles A. Bryan, Mr. Russell J. Egbert, Mr. Charles D. Lustfield, Mr. Rollin T. Sandberg, Mr. David G. Weinman.

Auburn University: Mr. James N. Issos, Mr. Marvin R. Reed, Mr. Kenneth E. Whipple.

Brigham Young University: Mr. Gurcharan S. Gill, Mr. John C. Higgins, Mr. Paul K. Merrill.

University of British Columbia: Mr. Earle P. Botta, Mr. Walter D. Burgess, Mr. Kim L. Chew, Mr. Lucien E. N. Delbrouck, Mr. John Froese, Mr. John R. Henderson, Mr. Gary J. Lastman, Mr. Denis K. Sjerve.

Brooklyn College: Mr. Leon E. Gerber, Mr. Larry Smith. 
Brown University: Professor Frederic C. Bisshopp, Mr. David Bell, Mr. John E. Brothers, Mr. Alan Howard, Mr. John M. Marstrand, Mr. Bernard V. O'Neill, Jr., Mr. William H. Reid.

Bucknell University: Mr. Gregory Wulczyn.

University of California, Berkeley: Mr. Leo H. Amaral, Mr. Robert E. Bradford, Mr. Barry W. Brown, Mr. Robert D. Brown, Mr. Charles H. Clemens, Mr. Hugh R. Coomes, Mr. Howard L. Dachslager, Mr. Charles S. Fisher, Mr. Francis J. Flanigan, Mr. Herbert J. Bauer, Mr. Eugene A. Herman, Mr. Peter G. Hinman, Mr. Edward A. Leeper, Mr. James F. McClendon, Mr. Leonard E. Ross, Mr. Malcolm J. Sherman, Mr. Seymour Singer, Mr. Alfred S. Tang, Mr. John J. Ucci.

University of California, Davis: Mr. Jim L. Chrislock, Mr. Ben J. Solomon.

University of California, Los Angeles: Mr. Takeo Akasaki, Mr. Alexander Hurwitz, Mr. Keith M. Kendig, Mr. Robert Maltz, Mr. Lewis C. Robertson, Mr. Frederick B. Strauss, Mr. Joel J. Westman.

University of California, Riverside: Mr. Clifford W. Arnquist, Mr. Donald E. McLeod, Mr. Scotty R. Neal.

University of California, Santa Barbara: Mr. Charles E. Huff, Mr. Robert K. Newcomb.

Calvin College: Mr. Robert J. Daverman, Mr. Sanford C. Leestma, Mr. John A. Veldhuis.

Case Institute of Technology: Mr. Madhukar V. Joshi, Mr. Harvey S. Price.

Catholic University of America: Miss Louise V. Arekelian, Mr. George Gebel, Mr. Edward J. O'Neill.

University of Chicago: Mr. Chao P. Chang, Mr. Walter Daum, Mr. Samuel M. Gedwiser, Mr. Robion C. Korby, Mr. Richard G. Larson, Mr. Gerson L. Levin, Mr. John L. MacDonald, Mr. Wolmer V. Vasconcelos.

University of Colorado: Mr. Kasturi L. Arora, Mr. Marvin C. Wunderlich.

Columbia University: Mr. Bernard M. Berlowitz, Mr. William T. England, Mr. Erhard R. Fernholz, Mr. Joseph L. Johnson, Mr. Jon A. Reed, Mr. Frank J. Servedio, Mr. Richard Sussman, Mr. Philip D. Wagreich, Mr. Sherwood Washburn.

University of Connecticut: Mr. Stephen A. Cutler, Mr. Ronald M. Gale, Mr. Arnold T. Iles, Mr. Roy F. Jordon, Miss JoAnn G. Pucilauskas.

Cornell University: Mr. Bruce A. Barnes, Mr. Graham C. Driscoll, Mr. James W. Graves, Mr. Joseph G. Rosenstein, Mr. Richard E. Schwartz, Miss Linda R. Sons, Mr. Barry Wolk.

Dartmouth College: Mr. David A. Gay, Miss Charlotte M. Foster, Mr. Edward P. Shaughnessy.

De Paul University: Mr. Louis E. Aquila, Mr. Constantine E. Georgakis, Mr. Alfonzo A. Patricelli.

Duke University: Mr. Stephan R. Cavior, Mr. Robert L. Ellis, Mr. Robert G. Van Meter.

Eastern Illinois University: Mr. D. Ferrel Atkins, Mr. Alphonso J. DiPietro.

Eastern New Mexico University: Professor Francis L. Buchanan.

Florida State University: Mr. Joe Neggers, Mr. Stanley E. Payne, Mr. James R. Retherford, Mr. Edward F. Wishart.

University of Florida: Mrs. Marijo O. LeVan, Mr. Reverdy E. Wright.

Frederick College: Mr. Stanley E. Marto, Jr., Mr. Samuel Reynolds.

University of Georgia: Mr. Harvey R. Durham, Mr. Karl R. Gentry, Mr. Sam B. Nadler, Jr., Mr. Choon-Jai Rhee.

Harvard University: Mr. Alberto De Carvalho Peizoto De Azevedo, Mr. Peter A. Belmont, Mr. Lawrence W. Conlon, Mr. Irving M. Isaacs, Mr. William B. Jones, 
Mr. John P. Labute, Mr. Stephen Lichtenbaum, Mr. Joseph Lipman, Mr. Alan G. Waterman.

Howard University: Mr. Jagjit S. Bakshi, Miss Marion L. Edinburgh.

Idaho State College: Mr. Walter James Dillon, Mr. Stephen Y. Fung, Miss Nadine J. Hanson.

University of Idaho: Miss Monika V. Aumann, Mrs. Ya-Yen L. Wang.

University of Illinois: Mr. Simmie S. Blakney, Miss Dorothy A. Bollman, Mr. Edmond

E. Granirer, Mr. Wolfgang Haken, Mrs. Ann H. Ihrig, Mr. Jingyal Pak, Mr.

James F. Phelan, Mrs. Lena C. Pu, Mr. Irvine J. Robinson, Mr. Douglas H. Taylor, Mr. Takesi, Watanabe.

Illinois Institute of Technology: Mr. Arthur I. Fine, Mr. Chester E. Tsai.

Indiana University: Mr. James T. Parr.

Institute for Advanced Study: Mr. Heisuke Hironaka, Mr. Yozo Matsushima, Mr. Sigfried Moran, Mr. David B. Mumford, Mr. Hideo Shimizu, Mr. Brian F. Steer.

Iowa State University: Mr. Gary L. Anderson, Mr. Kenneth L. Deckert, Mr. John N. Mordeson.

State University of Iowa: Mr. John S. Bradley, Mr. Robert J. Gregorac, Mr. J. Colby Kegley, Mr. Gerald L. Kerr, Mr. Lloyd L. Lininger, Mr. Albert D. Otto.

University of Kansas: Mr. Richard C. Basinger, Mr. Emanuel G. Calys, Mr. William M. Causey, Mr. Charles R. Combrink, Mr. Wilfred M. Greenlee, Mr. Larry F. Heath, Mr. Thomas J. Henninger, Mr. Buddy A. Johns, Jr.

Kent State University: Mr. Marion H. Amick, Mr. Paul A. Hulka, Mr. James B. Thomas.

Lehigh University: Mr. Jerry D. Badger, Mr. Cheung Y. Keung, Mr. Murray R. Kirch, Mr. Robert J. McNelis, Mr. Dale F. Oexmann, Mr. Andrew K. Snyder.

Le Moyne College: Mr. Frederick J. Carter, Professor Thomas S. Frank.

Louisiana State University: Mr. Robert M. Brooks, Mr. Dennison R. Brown, Mr. Charles J. Feaux, Mr. Kenneth O. Leland.

Loyola College: Mr. Bernard J. Weigman, Jr.

Marquette University: Mr. Thomas M. Mansheim.

University of Maryland: Mr. Derrill J. Bordelon, Miss Kathy K. Forbes, Mr. Richard Freiman, Mr. Cephas D. Hughes, Mr. Addul R. Kiwan, Mr. Simon A. Levin, Mr. Anastosios Mallios, Mr. Howard P. Rawlings, Mr. Philip W. Schaefer, Mr. Frank W. Wilson, Jr.

University of Massachusetts: Mr. John O. Hebert, Mr. Royce D. Purington, III, Mr. Vaithilingam Thuraisamy.

Massachusetts Institute of Technology: Mr. Michael A. Arbib, Mr. Alfred Clark, Jr., Mr. Donald A. Dawson, Mr. Bert E. Fristedt, Mr. Morton M. Hackman, Mr. Ulrich Hirzebruch, Mr. David F. Isles, Mr. Robert W. Johnson, Mr. Geoffrey A. Kandell, Mr. Nick Metas, Mr. Peter D. Rosenbaum, Mr. Werner G. Schaal, Mr. David I. Schneider, Mr. John H. Smith, Mr. Norton Starr.

McMaster University: Mr. Hoshang P. Doctor, Mr. Siegfried L. Jansen, Mr. Donald J. Miller.

University of Miami: Mr. John E. Dennis, Mr. Robert T. Ramsay, Mr. Robert Sudakow.

Michigan State University-East Lansing: Mr. Preston B. Britner, Mr. Kwan-Wei Chen, Mr. Merle D. Guay, Mr. Somashakhar A. Naimpally, Mr. Richard P. Osborne.

Michigan State University-Oakland: Mr. Steven L. Hunt, Mr. Eugene LaLowe, Mr. Carl J. Mabee.

University of Michigan: Mr. Alan E. Farley, Mr. Robert M. Fossum, Mr. Henry H. Glover, Mr. King M. Lee, Mr. William F. Lucas, Mr. Jiang Luh, Mr. Eric A. Nordgren, Mr. George F. Votruba. 
University of Minnesota: Mr. Kenneth R. Berg, Mr. Roger S. Countryman, Jr., Mr. Larry C. Grove, Mr. James K. Foster, Mr. Lawrence J. Lardy, Mr. Boas Natzitz, Mr. Glenn E. Schober, Mr. Gaylord P. Schwartz.

University of Missouri: Mr. Frederick W. Wilke.

Monmouth College: Professor John D. Arrison.

University of Nebraska: Mr. Paul L. Dussere, Mr. Terry L. Jenkins.

University of New Hampshire: Mr. Amory A. Aldrich, Jr., Mr. David E. Hilt, Mr. Raymond U. Stebbins.

University of Nevada: Mr. Maurice C. Devore, Miss Eileen L. Halligan, Mr. John C. Miller.

New Mexico State University: Mr. J. Mack Adams, Mr. Howard E. Lacey, Mr. Edmund J. Peake, Jr.

New York University: Mr. Hector O. Fattorini, Professor Yukio Kusunoki, Mrs. Joan G. Landman, Mr. Pedro Nowasad, Mr. Edmund Pribitkin, Professor Madan L. Puri, Mrs. Lesley M. Sibner, Mr. Marion S. Weiner, Mr. Raymond O. Wells, Jr.

University of North Carolina: Mrs. Carole M. Adams, Mr. William L. Bynum, Mr. Hughes B. Hoyle, III, Mr. Phillip M. Kannan, Mr. Curtis L. Outlaw, Mr. Nelson F. Page.

North Texas State University: Mr. William E. Milner, Mr. Darwin E. Peek, Mr. Floyd R. Vest.

Northwestern University: Mr. Carl L. DeVito, Mr. Helmut P. Epp, Miss Susana E. Gastaminza, Mr. Lawrence L. Larmore, Jr., Mr. Philip A. Ostrand, Mr. Dario Juan Picco.

Old Dominion College: Mr. Bill J. Georges.

Ohio Wesleyan University: Mr. Fred M. Haney, Mr. Wilfred J. Hansen, Miss Leslie S. Smith.

Oklahoma State University: Mr. Melvin Roy Hagan, Mr. Robert R. Kinkade, Professor Robert L. Tennison.

University of Oklahoma: Mr. John B. Bennett, Mr. Marc Q. Jacobs, Mr. Leo J. Pratte.

University of Oregon: Mr. Arne P. Baartz, Mr. Richard B. Crittenden, Mr. Frank R. DeMeyer, Mr. Roger M. Fischler, Mr. Yu-Lee Lee, Mr. Robert J. Lindahl, Mr. James D. Stafney.

Pennsylvania State University: Mr. Anthony W. Hager, Mr. Robert C. Moore, Mr. Luis E. Sanchis, Mr. David P. Stadtlander, Mr. John H. Ursell, Mr. Earl R. Willard.

University of Pittsburgh: Mr. Edward H. H. Gade, III, Mr. Franz X. Hiergeist, Mr. James W. Salter.

Polytechnic Institute of Brooklyn: Mr. Paul I. Feder, Mr. Stewart G. Nagler, Mr. Anthony J. Naro.

Princeton University: Mr. Anthony W. Knapp, Mr. Kee Y. Lam, Mr. Henry R. Mitchell, Mr. Robert T. Moore, Mr. Anthony V. Phillips, Mr. William M. Priestley, Mr. Daniel Reich, Mr. Joel W. Robbin, Mr. Lawrence C. Siebenmann, Mr. Neville F. Smythe, Mr. Jonathan D. Sondow, Mr. Charles E. Stenard, Mr. Gilbert W. Stewart, III, Mr. John B. Wagoner, Mr. Benjamin Weiss.

Purdue University: Mr. Ralph J. Faudree, Mr. Eugene M. Friedman, Mr. Donald L. Prullage, Mr. Francis D. Ryan, Mr. Larry E. Snyder, Mr. Benjamin A. Trimble.

Queens College: Mr. Bernard R. Bernstein, Mr. Henry T. Harris, Miss Alice G.Ringel.

Rensselaer Polytechnic Institute: Mr. Bruce E. Goodwin, Mr. Sherman Tsao.

University of Rhode Island: Mr. James J. Foster, Mr. John T. James, Mr. Manuel Renasco.

Rosary Hill College: Miss Lois A. Grabenstatter, Mr. Robert G. McGee, Mr. Joseph A. Orzechowski. 
Rutgers, The State University: Mr. Joseph Barback, Mr. William H. Caldwell, Mr. Vincent J. Mancuso, Mr. Angelo Pelios.

Sacramento State College: Mr. Byron D. Hendrix, Mr. Harvey J. Bader, Mr. Barthel W. Huff.

San Diego State College: Mr. Ralph O. Mann, Mr. Froylan Tiscareno, Mr. Herman H. Wong.

South Dakota School of Mines and Technology: Mr. Gorman R. Nelson, Mr. Wayne Walther.

Southern Illinois University: Mr. Jerry K. Cline, Mr. David W. Kammler, Mr. Chull Park.

University of Southern California: Mr. Loren D. Enochson, Mrs. Helen S. Lim, Mr. Leland Sapiro, Mr. Thos. F. Storer.

Stanford University: Mr. James A. Anderson, Mr. Paul C. Chestnut, Mr. Gerald S. Goodman, Mr. David Hemmendinger, Mr. John C. Heywood, Mr. Rodney W. Johnson, Mr. Eugene T. Y. Lee, Mr. Frederick W. Luttmann, Mr. Thomas A. McCready, Mr. Peter Marks, Mr. Edward V. Mirmak, Mr. Frank W. Owens, Mr. W. Stephen Piper, Mr. Ernst J. P. George Schmidt, Mr. Jon T. Schnute, Mr. Andrew Y. Schoene, Mr. William J. Sweeney, Mr. Charles C. Wong.

Stephen F. Austin State College: Mr. Glenn E. Johnston, Mr. Joseph T. Robbins.

Stevens Institute of Technology: Mr. Peter M. Brady, Jr., Mr. Rudolph H. Downs, Mr. David B. Hill.

University of Tennessee: Mr. Don B. Hinton, Mr. Donald R. LaTorre, Mr. Richard L. Robinson, Mr. Donald G. Stewart, Mr. Anton J. Zettl.

University of Texas: Mr. Thomas A. Atchison, Miss Barbara A. Clinger, Mr. Hadi Haddad, Mr. John K. Perryman, Mr. Charles D. Robinson, Mr. Sam W. Young.

University of Toronto: Mr. Walter P. Kotorynski, Mr. Urs R. Maag, Mr. Andre Plante, Mr. Kenneth S. Williams.

Trinity College, Connecticut: Mr. Thomas R. Berger, Mr. Raymond A. Drate, Mr. Richard O. Hill, Jr.

Tulane University: Mr. William W. Babcock, Mr. Robert Bartoszynski, Mr. William M. Boyce, Mr. Mohammad R. Haque, Mr. Stanislaw K. Knapowski.

University of Tulsa: Mr. Steve K. Atiyah, Mr. John W. Rainey, Mr. Robert E. South.

Union College: Professor H. Kendrick Holt, Professor Ingo Maddaus, Jr., Professor William C. Stone.

University of Utah: Mr. Ronald D. Jamison, Mr. Thomas L. Sherman, Mr. Willes L. Werner.

Vanderbilt University: Mr. Donald F. Bailey, Mr. Oscar T. Nelson, Jr., Mr. Richard $\mathrm{K}$. Williams.

University of Washington: Mr. Laurence W. Baggett, Mr. Donald R. Beldin, Mr. Carlos R. Borges, Mr. Oscar M. Browne, III, Mr. George E. Denzel, Mr. David M. Eaves, Mr. Palaniappan Kannappan, Mr. Michael S. Martin, Mr. Ray Mines, Mr. Keith L. Phillips, Mr. Lawrence J. Pozsgay, Mr. Galen J. Roush, Mr. Chung-Tuo Shih, Mr. Robert W. Stringall, Miss Eileen L. Ting.

Washington State University: Mr. Theodore A. Burton.

Washington University: Mr. Kenneth I. Gross, Mr. Frank R. Singer, III, Mr. Philip B. Zwart.

Wayne State University: Mr. Hans-Heinrich W. Herda, Mr. Ranganathan Srinivasan. Wesleyan University: Mr. Ronald V. Book, Mr. William O. Dixon, Miss Patricia F. McHugh. 
Western Michigan University: Miss Suzanne H. Arns, Mr. Richard H. Reese, Mr. James E. Riley.

Western Washington State College: Mr. Sanford Berman, Mr. Donald W. Foster, Mr. Ronald J. Saltis.

Williams College: Mr. John L. Pfaltz.

College of William and Mary: Mr. Willard R. Boland, Mr. Hugh B. Easler, Mr. Robert J. Hursey, Jr., Mr. Michael H. Kutner, Mr. Nathaniel R. Withers, Mr. George R. Young.

University of Wisconsin-Madison: Mr. David F. Appleyard, Mr. Robert Barnhill, Mr. Gerald L. Brown, Mr. Jerome Dancis, Mr. Neil A. Davidson, Mr. Rolfe P. Ferguson, Mr. Daniel P. Giesy, Mr. Richard M. Harper, Mr. David W. Henderson, Mr. Alexander Herzog, Mr. Victor E. Hill, Mr. Fred C. Icard, Jr., Mr. Gerald J. Janusz, Mr. Carl E. Jukkola, Mr. Samuel D. Lawn, Mr. William A. Lopez, Mr. John R. Michel, Mr. James D. Moore, Mr. James A. Murtha, Mr. Dwight M. Paine, Mr. Daniel G. Rider, Mr. Dennis E. Smith, Mr. Richard B. Thompson, Mr. David W. Walkup, Mr. Julian Weissglass, Mr. Thomas Perrin Wright, Jr., Mr. Robert L. Wilson, Jr., Mr. Julius M. Zelmanowitz.

University of Wisconsin-Milwaukee: Mr. Samuel L. Dunn, Miss Edwardine M. Geniusz, Mr. Marvin L. Weber.

Yeshiva University: Mr. Robert A. Bagnato, Mr. Jerome Blau, Mr. Aaron Lebowitz, Mr. Louis Raymon, Mr. Edwin J. Sherry, Mr. Hyam L. Zuckerberg.

Yale University: Mr. George A. Anderson, Mr. Richard W. Beals, Mr. Peter C. Greiner, Mr. Gerard J. Schellekens, Mr. Dominic C. Soda.

It was reported that two persons were elected to membership as Institutional Associates: RIAS, Mr. Nam P. Bhatia, Mr. Carlo Perello.

The following Presidential appointments were reported:

As a Committee to Select the Gibbs Lecturer for 1965 and 1966: A. H. Taub, Chairman, R. C. Buck, R. J. Duffin.

As an Organizing Committee for the 1964 Summer Institute on Algebraic Geometry: Oscar Zariski, Chairman, W. L. Chow, Maxwell Rosenlicht, D. C. Spencer, John Tate.

As representatives of the American Mathematical Society at the Conference in Washington on the Gilliland Report: Bernard Friedman, W. T. Martin, G. W. Mackey, P. T. Bateman, P. R. Halmos.

As a Committee to Award the Veblen Prize: S. S. Chern, S. Eilenberg, R. H. Bing.

The Secretary reported that the following persons had accepted invitations to deliver hour addresses at meetings of the Society: Gerald R. MacLane and Walter L. Baily at Chicago, April, 1963. F. B. Wright at Georgia Institute of Technology, November, 1963. Kai Lai Chung at California Institute of Technology, November, 1963. Marshall Hall and George B. Dantzig at Reno, Nevada, April, 1964. Lars Onsager at Miami in January, 1964, Gibbs Lecturer. 
The Secretary reported that the following members represented the Society: R. P. Agnew, at the inauguration of W. Allen Wallis as President of the University of Rochester; Mina Rees, at the inauguration of Rosemary Park as President of Barnard College; W. E. Byrne, at the inauguration of Thomas Marshall Hahn, Jr., as President of Virginia Polytechnic Institute; Elliott L. Buell, at the inauguration of Clifton Woodford Emery, Jr., as President of Worcester Junior College; Donald W. Blackett, at the Centennial Convocation at Boston College.

The Secretary reported that the Council voted, on recommendation of the Organizing Committee for Summer Institutes, to hold a Summer Institute in 1964 on Algebraic Geometry, under the Chairmanship of Professor Oscar Zariski. It also voted on the recommendation of the Committee on Applied Mathematics to hold a Symposium on Nonlinear Partial Differential Equations in Mathematical Physics at a Spring 1964 meeting of the Society, under the chairmanship of Professor Robert Finn.

The Council received the following motion from the Board of Governors of the Mathematical Association of America: "The MAA in considering these and other pressing problems of graduate programs in mathematics, including, in particular, those mentioned in the Report of the President's Science Advisory Committee, Report Number One: Graduate Training in Engineering, Mathematics, and Physical Sciences, published by the White House, December 12, 1962, approved the following motion: 'The MAA shall invite the AMS to join in establishing a joint committee to consider the graduate program in mathematics in the broadest sense. This committee shall concern itself with education in theoretical and applied mathematics. We propose the establishment of this committee because of pressing problems involving the quantity of graduate students in mathematics and the needs of educational institutions and industry for more adequately trained mathematicians. If this committee wishes to enlist the opinions or cooperation of other groups, such as the Conference Board of the Mathematical Sciences, it should feel at liberty to do so.' "The proposal was accepted by the Council and the President authorized to proceed with the appointment of a joint committee.

JoHN W. GREeN

Secretary

Everett Pitcher

Associate Secretary 